Einladung zum Beitritt zu einer Leonhard Euler-Gesellschaft.

Im September 1909 hat die Schweizerische Naturforschende Gesellschaft zu Lausanne den BeschluB gefaßt, die gesamten Werke Leonhard Eulers herauszugeben and hierdurch einen wiederholt und dringend geäuberten Wunsch der ganzen mathematischen Welt zu erfüllen. Eulers auBerordentlich vielseitige Arbeiten haben das fast einzigartige Schicksal, nicht zu veralten; sie bilden auch heute noch, nachdem der 100 jährige Todestag des gefeierten Mathematikers schon hinter ans liegt, eine bei weitem nicht erschöpfte Quelle für wissenschaftliche Forschung und Erkenntnis. Viele Arbeiten Eulers sind in heute sehr selten gewordenen Zeitschriften des 18. Jahrhunderts verborgen, and so war der Wunseh, alles, was vọn Eulers Hand stammt, leicht zugänglich und übersichtlich geordnet zu besitzen, ein in der Tat durchaus berechtigter.

Die vorläufige Berechnung des Cmfangs einer Gesamtausgabe der Eulerschen Werke schätzte die Anzahl der hierfür nötigen Bände auf 40 bis 45 , und auf dieser Basis erfolgte die Finanzierung des Unternehmens. Die Gesamtkosten wurden auf ungefähr eine halbe Million Franken berechnet, welche Summe durch Abonnemente und durch freiwillige Beiträge gedeckt erschien.

Bis jetzt sind neun Bände herausgegeben worden, welche in jeder Hinsicht, sowohl was die kritische Durcharbeitung, als anch, was die Schönheit des Druckes betrifft, überall ungeteilten Beifall gefunden haben. Der zehnte ist im Erscheinen begriffen. Allein es hat sich dabei leider herausgestellt, daB die Kosten höhere sind, als angenommen war, soda $B$ trotz unseren 362 Abonnenten diese nean Bände (Abonnementspreis per Band 25 Fr.) ein Defizit von ca. 57000 Franken mit sich gebracht haben, welches aus dem Euler-Fonds gedeckt werden mubte. Dieser Fonds, gestiftet durch Beiträge von Behörden, wissenschaftlichen und industriellen Gesellschaften und Privatpersonen, ist heute schon auf 84000 Fr. zusammengeschmolzen. Hierzu kommt, daB die angenommene Zahl der Bände nicht ausreichen wird, um sämtliche Werke des fast unerschöpflichen Gelehrten aufzunehmen, wenn man sio nicht will ungebührlich anschwellen lassen. Die Kaiserl. Akademie der Wissenschaften ron St. Petersburg hat eine groBe Zahl bisher unbekannter Manuskripte der Euler-Kommission zax Verfügung gestellt, Briefe Eulers kommen überall zum Vorschein; aber auch die bereits im Druck erschienenen Arbeiten Eulers nehmen in der 
neuen Ausgabe, namentlich infolge der nachträglich gewählten, dem monumentalen Charakter eines solchen Werkes besser entsprechenden größeren Schrift, sowie wegen der Vorreden und der unumgänglich notwendigen Anmerkungen der Herausgeber usw. einen bedeutend breiteren Raum ein, als anfänglich war angenommen worden.

So stehen wir heute vor der unerfreulichen Tatsache, daß die Gesamtkosten fast das Doppelte der ursprünglichen Schätzung betragen werden, nämlich ungefähr 900000 Franken, und unser voraussichtliches Defizit wird vermutlich die Höhe von ca. $200000 \mathrm{Fr}$. erreichen, da eine Erhöhung des Bandpreises wegen der gegenüber den Abonnenten eingegangenen Verpflichtungen ausgeschlossen ist.

Wenn die Euler-Kommission der Schweizerischen Naturforsehenden Gesellschaft trotzdem den Mat nicht verliert, die Riesenaufgabe zu einem glücklichen Ende zu führen, so entspringt diese Zuversicht der Überzengung, daB damit etwas wahrhaft GroBes und Nützliches geschaffen wird, und sie wurzelt in der festen Hoffnung, daB es an Freunden nicht fehlen werde, die dem Unternehmen tatkräftige Hilfe zu leisten gesonnen sind.

In dieser Überzeugung haben wir beschlossen, für die Dauer der Herausgabe der Eulerschen Werke (circa 15 Jahre) eine Leonhard EulerGesellschaft ins Leben zu rufen, deren Mitglieder sich zu einem Jahresbeitrag von wenigstens $10 \mathrm{Fr}$. verpflichten. Die Mitglieder werden jährlich einen kurzen Bericht über den Stand der Herausgabe erhalten; es sollen ihnen auch sukzessive die verschiedenen Porträts, die von Euler vorhanden sind, in guten Reproduktionen als Dank zugestellt werden.

So hoffen wir denn, daß nicht nur die Mathematiker, sondern auch zahlreiche Freunde der Wissenschaft überhaupt, in- und anßerhalb der Schweiz, unserer Einladung zum Beitritt Folge leisten werden, und daB namentlich auch die mathematischen, physikalischen und astronomischen Gesellschaften, die Ingenieurvereine, die Versicherungsgesellschaften und die großen industriellen Unternehmungen, die sich auf den mathematischen Wissensehaften aufbauen, sich als Kollektirmitglieder anschließen werden, damit das gewaltige Denkmal, das einem der größten Gelehrten aller Zeiten errichtet werden soll, nicht ein Strickwerk bleibe, sondern fertig ansgebaut werden könne, zum dauernden Ruhme Eulers und zur Ehre und Förderung der mathematischen Wissenschaften.

\section{Fritz Sarasin.}

Ferdinand Rudio.

\section{Eduard His-Schlnmberger.}

Basel und Zürich, im November 1913.

Die Beitrittserklärung ist zu senden an den Schatzmeister der Euler-Kommission, Herrn Ed. His-Sehlumberger, Aeschenvorstadt 15, Basel. 